\title{
BMJ Open Public health impact model estimating the impact of introducing an adjuvanted recombinant zoster vaccine into the UK universal mass vaccination programme
}

\author{
Desirée A M van Oorschot, ${ }^{\oplus 1}$ Manjit Hunjan, ${ }^{2}$ Benjamin Bracke, ${ }^{1}$ \\ Stéphane Lorenc, ${ }^{3}$ Desmond Curran, ${ }^{1}$ Helen Starkie-Camejo ${ }^{2}$
}

To cite: van Oorschot DAM, Hunjan M, Bracke B, et al. Public health impact model estimating the impact of introducing an adjuvanted recombinant zoster vaccine into the UK universal mass vaccination programme. BMJ Open 2019;9:e025553. doi:10.1136/ bmjopen-2018-025553

- Prepublication history and additional material for this paper are available online. To view these files, please visit the journal online (http://dx.doi. org/10.1136/bmjopen-2018025553).

Received 30 July 2018

Revised 15 January 2019

Accepted 27 February 2019

Check for updates

(C) Author(s) (or their employer(s)) 2019. Re-use permitted under CC BY-NC. No commercial re-use. See rights and permissions. Published by BMJ.

${ }^{1}$ GSK, Wavre, Belgium

${ }^{2}$ GSK, Uxbridge, UK

${ }^{3}$ Freelance on behalf of GSK, Wavre, Belgium

\section{Correspondence to} Desirée A M van Oorschot; desiree.x.van-oorschot@gsk. com

\section{ABSTRACT}

Objectives In 2013, the herpes zoster (HZ) immunisation programme was introduced in the UK, recommending vaccination of adults 70 years of age (YOA) with the zoster vaccine live $(\mathrm{ZVL})$, the only vaccine available at the time. The recently approved adjuvanted recombinant zoster vaccine (RZV) has a substantially different clinical profile that may offer additional benefits. This study aimed to 1) assess the public health impact (PHI) of introducing RZV in the UK compared with the current vaccination strategy and 2) explore via scenario analyses the optimal age group of vaccination in terms of $\mathrm{PHI}$.

Design A previously developed health economic model was adapted to the UK setting.

Setting Calculations were based on efficacy data from pivotal clinical trials, $\mathrm{HZ}$ incidence and postherpetic neuralgia (PHN) probability from a UK study and HZassociated complication rates from published literature. Population The base-case population considered a 2018-projected UK vaccination cohort of individuals 70 YOA.

Interventions Vaccination with ZVL or RZV, assuming a first-dose coverage of $48.3 \%$ for both vaccines and $70 \%$ compliance for the second dose of RZV.

Outcome measures Outcomes included reduction of $\mathrm{HZ}$ and PHN cases, complications and the use of healthcare resources over a life-time horizon. The impact of coverage and second-dose compliance was also explored.

Results Compared with no vaccination, RZV would lead to a reduction of $30262 \mathrm{HZ}$ and $5409 \mathrm{PHN}$ cases while ZVL would lead to a reduction of $7909 \mathrm{HZ}$ and $3567 \mathrm{PHN}$ cases. The number needed to vaccinate to prevent $1 \mathrm{HZ}$ case is 12 with RZV and 45 with ZVL. The highest PHI with RZV could be achieved in individuals 60 or 65 YOA. Conclusion Under the model assumptions, RZV is predicted to avert more $\mathrm{HZ}$ and $\mathrm{PHN}$ cases compared with ZVL. Results were robust under different scenario and sensitivity analyses.

\section{INTRODUCTION}

The varicella zoster virus (VZV) usually affects children and leads to varicella, also known as chickenpox. The virus remains dormant lifelong in patients' dorsal root ganglia. ${ }^{1}$ Later
Strengths and limitations of this study

- The most recent UK-specific data from published literature is included in the ZOster ecoNomic Analysis model.

- Model structure and inputs have been validated by external experts.

- Results of this analysis estimates the impact of a recombinant zoster vaccine (RZV) programme in the UK population in 2018.

- Further analyses have to be performed once longterm effectiveness data become available on the duration of protection of RZV.

- Assumptions regarding second-dose compliance had to be made in absence of real-world data.

in life, VZV-specific T-cell immunity decreases due to immunosenescence or immunosuppressing illnesses or medications. Reactivation of VZV results in herpes zoster (HZ), also called shingles. ${ }^{23}$ Over $95 \%$ of individuals will have acquired VZV during their childhood or early adulthood. ${ }^{45}$ Approximately one in three people will develop HZ during their lifetime with the risk increasing sharply after the age of 50 years of age (YOA), leading to an estimated $5 \mathrm{HZ}$ episodes per 1000 people in the UK, each year. ${ }^{6-8}$ Similar incidence rates were reported in other European countries and elsewhere. ${ }^{2} 7$ Furthermore, results from observational studies suggest that $\mathrm{HZ}$ incidence has risen during the past decade in various countries and is predicted to continue to rise as the average age of the population increases. $^{2910}$

$\mathrm{HZ}$ tends to start with prodromal pain, followed by a dermatomal rash which is usually unilateral and develops typically over the trunk or face. Rash is often accompanied by severe pain. Skin lesions and pain usually disappear completely within 4-6 weeks. Postherpetic neuralgia (PHN), often defined 
as pain persisting or appearing 30-90 days after rash onset, is the most common complication which can last from several weeks to months. ${ }^{811}$ Even though mortality due to $\mathrm{HZ}$ infection is low, $\mathrm{HZ}$ greatly affects quality of life (QoL) in terms of physical and social functioning and the well-being of the patients. ${ }^{12}$ Furthermore, severity of pain strongly correlates with the reported QoL. ${ }^{11}{ }^{13}$ Current treatment options, which mainly rely on antivirals, analgesics and antidepressants, provide only partial symptomatic relief and limited protection against the development of PHN and other complications. Thus, the impact of the disease on patients' QoL is not adequately managed with existing interventions. ${ }^{11}$

In the UK, the Joint Committee on Vaccination and Immunisation (JCVI) recommended universal mass vaccination (UMV) for $\mathrm{HZ}$ using zoster vaccine live (ZVL; Zostavax),${ }^{14}$ the only vaccine available at the time the UMV programme was introduced in 2013. ZVL is a live-attenuated virus vaccine indicated for the prevention of $\mathrm{HZ}$ and, in Europe, of PHN in individuals $\geq 50 \mathrm{YOA} .{ }^{15}$ Vaccine efficacy (VE) against $\mathrm{HZ}\left(\mathrm{VE}_{\mathrm{HZ}}\right)$ of $\mathrm{ZVL}$ in the shingles prevention study (SPS) was $63.9 \%$ in individuals $60-69$ YOA and $37.6 \%$ in individuals $\geq 70$ YOA. $^{15}{ }^{16}$ Long-term clinical trial data and observational effectiveness studies showed that VE of ZVL decreased substantially over time conferring little or no protection against HZ beyond 8 years after vaccination. ${ }^{1718}$

Even though ZVL is indicated in individuals $\geq 50 \mathrm{YOA}$, the JCVI recommended vaccination with ZVL at $70 \mathrm{YOA}$ (and a catch-up vaccination for people $78 \mathrm{YOA}$ ), based on clinical trial data and an economic model showing that vaccination at $70 \mathrm{YOA}$ would be the most cost-effective option given that the burden of disease increases with age, while VE of ZVL decreases in older individuals and over time. ${ }^{314}$ A further limitation to the indicated use of ZVL in individuals $\geq 50 \mathrm{YOA}$ is its contraindication in primary or acquired immunodeficiency states due to blood disorders or other types of cancer, infection with HIV or due to high-dose immunosuppressive therapy. ${ }^{15} 19$ A proportion of individuals would therefore not be able to receive ZVL. ${ }^{20}$

A novel adjuvanted recombinant zoster vaccine (RZV, Shingrix) has been granted marketing authorisation by the European Medicines Agency (EMA) and is indicated for use in individuals $\geq 50 \mathrm{YOA}$. RZV is a non-live vaccine consisting of the VZV glycoprotein $\mathrm{E}$, a prominent antigen target of VZV-specific CD4+ T-cell immune responses, and AS0 $1_{\mathrm{B}}$ adjuvant system, which boosts immunogenicity and duration of the immune response. ${ }^{21} \mathrm{RZV}$ is administered in two doses 2-6 months apart. Because RZV is a non-live vaccine, it is not contraindicated in immunocompromised (IC) individuals. While at this point in time, there is only limited data available regarding the use of Shingrix in subjects with confirmed or suspected immunosuppressive or immunodeficient conditions, further studies are ongoing. As with other vaccines, the administration of Shingrix to IC subjects should be based on careful consideration of potential benefits and risks. ${ }^{22}$ Two large, phase III trials, that is, the Zoster Efficacy Studies in Adults 50 and 70 YOA or older (ZOE-50 [NCT01165177] and ZOE-70 [NCT01165229], respectively) demonstrated high $\mathrm{VE}_{\mathrm{HZ}}$ of RZV in all age groups; $\mathrm{VE}_{\mathrm{HZ}}$ was $97.2 \%$ in individuals $\geq 50 \mathrm{YOA}$ included in the ZOE-50 study and $91.3 \%$ in individuals $\geq 70 \mathrm{YOA}$ included in the ZOE- 50 and ZOE-70 studies. ${ }^{23}{ }^{24} \mathrm{VE}$ persisted over the 4-year duration of the clinical trial. ${ }^{24}$

The objective of this study is to explore the public health impact of introducing RZV in the UK in the routine population 70 YOA. The effect of RZV and ZVL on HZ and PHN incidence, complications and health resource utilisation is compared with no vaccination. Different scenario analyses are carried out to assess the impact of first-dose RZV coverage and second-dose RZV compliance and to determine the optimal age for vaccination.

\section{METHODS}

\section{Patient and public involvement}

Patients or public were not involved as the analysis is based on mathematical modelling.

\section{Model structure}

The ZOster ecoNomic Analysis (ZONA), a static multicohort Markov model previously developed using Microsoft Excel, was adapted to the UK setting. The economic model considers up to five various age cohorts that can transition between different health states, including no HZ, HZ, health states associated with complications of HZ (PHN and non-PHN complications) and death from $\mathrm{HZ}$ or natural causes. ${ }^{25}$ Cycle length is set to 1 year and follows all subjects from the year of intervention over their remaining lifetime. The model has three different arms, having the same yearly model structure: no vaccination, vaccination with RZV and vaccination with ZVL. Within the vaccine strategy, individuals can be fully compliant with the vaccine dosing schedule, only partially or not vaccinated at all (depending on the compliance rate). Further details regarding the model structure are reported in the online supplementary information (SI) figure 1 and in the study by Curran $e t a .^{25}$

\section{Model input parameters}

Wherever possible, UK-specific data were used. Efficacy data for RZV and ZVL were derived from pivotal clinical trials conducted for ZVL and RZV. ${ }^{16232426}$ Both model structure and global inputs such as VE and waning were validated with an external expert panel (epidemiologists, clinicians and health economists with a background in HZ) in September 2016.

\section{Demographics}

Populations in the model are projected to 2018 values. The base-case population consisted of the routine vaccination cohort 70 YOA. Based on projections by the Office of National Statistics (ONS), ${ }^{27}$ the predicted population 


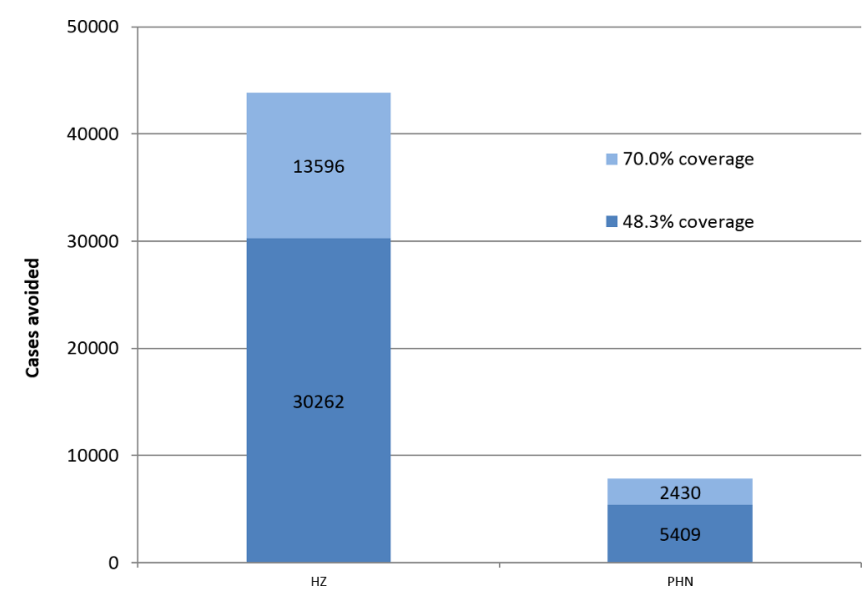

Figure 1 Impact of increasing RZV coverage to $70 \%$. Additional $\mathrm{HZ}$ and $\mathrm{PHN}$ cases avoided (light blue bars) comparing RZV vs no vaccination in people 70 YOA. Seconddose compliance for RZV was set to $70 \%$. HZ, herpes zoster; RZV, adjuvanted recombinant zoster vaccine; $P H N$, postherpetic neuralgia; YOA, years of age.

numbers in the routine cohort of $70 \mathrm{YOA}$ is 722616 , in 2018. Different age cohorts were modelled for use in scenario analyses (table 1 ).

All-cause mortality rates were derived from ONS data projected to the year $2018 / 2019$ (online SI table 1 ). ${ }^{27}$

\section{Epidemiology}

$\mathrm{HZ}$ incidence

HZ incidence was derived from a recent UK Clinical Practice Research Datalink (CPRD) study, which assessed the incidence of $\mathrm{HZ}$ in immunocompetent (IC-free) and IC individuals between 2000 and $2012^{28}$ (online SI table 2). The CPRD database study presents the most recent realworld data on $\mathrm{HZ}$ incidence and was therefore considered the most appropriate source for this parameter. The IC-free and IC population were matched by age, gender and location of general practitioner (GP) and the proportion of IC individuals was adjusted in the whole population to account for an increase in immunodeficiencies in older individuals. In the age group 70-79 YOA, 35\% of individuals had primary or acquired immunodeficiency and a subgroup of this IC population is contraindicated to receive ZVL. Incidence numbers were converted to annual probabilities of developing HZ (table 1). Lower and upper ranges of probabilities for $\mathrm{HZ}$ incidence in the whole population were obtained from published data since it was not possible to derive it from the split IC and IC-free data set analysed in the CPRD study ${ }^{3}$ (online SI table 3).

Incidence rate of recurrent $\mathrm{HZ}$ is assumed to be the same as the incidence of the initial event. This assumption is supported by published data which indicates that

\begin{tabular}{|c|c|c|c|c|c|c|}
\hline Age & & 50 YOA & 60 YOA & 65 YOA & 70 YOA & 80 YOA \\
\hline $\begin{array}{l}\text { Number of people } \\
\text { in age group in } \\
2018\end{array}$ & & 908255 & 783067 & 686215 & 722616 & 389107 \\
\hline \multirow{2}{*}{$\begin{array}{l}\text { HZ incidence per } \\
1000 \text { individuals }\end{array}$} & IC & 6.85 & 8.80 & 9.93 & 11.32 & 12.61 \\
\hline & IC-free & 4.9 & 6.92 & 8.62 & 11.04 & 11.02 \\
\hline $\begin{array}{l}\text { Proportion } \\
\text { developing PHN } \\
\text { (\%) }\end{array}$ & & 11.42 & 13.89 & 15.71 & 17.12 & 20.42 \\
\hline \multirow{4}{*}{$\begin{array}{l}\text { Non-PHN } \\
\text { complications } \\
\text { incidence (\%) }\end{array}$} & Ocular & 2.87 & 3.82 & 3.82 & 4.14 & 5.41 \\
\hline & Neurological & 2.46 & 3.17 & 3.17 & 5.99 & 4.23 \\
\hline & Cutaneous & 1.74 & 1.05 & 1.05 & 2.09 & 2.44 \\
\hline & Other & 2.03 & 1.63 & 1.63 & 2.44 & 2.85 \\
\hline \multirow{3}{*}{$\begin{array}{l}\mathrm{HZ} \text { vaccine } \\
\text { efficacy, \% } \\
\text { (range) }\end{array}$} & $\begin{array}{l}\text { RZV } \\
\text { Two doses }\end{array}$ & $98.4(95-100)$ & $98.4(95-100)$ & $98.4(95-100)$ & $97.8(94.1-100)$ & $97.8(94.1-100)$ \\
\hline & $\begin{array}{l}\text { RZV } \\
\text { One dose }\end{array}$ & $90.0(58.9-98.9)$ & $90.0(58.9-98.9)$ & $90.0(58.9-98.9)$ & $69.5(24.9-89.1)$ & $69.5(24.9-89.1)$ \\
\hline & ZVL & $69.8(54.1-80.6)$ & $63.9(56.0-71.0)$ & $63.9(56.0-71.0)$ & $40.85(28.0-52.0)$ & $18.25(0-48.0)$ \\
\hline \multirow{3}{*}{$\begin{array}{l}\text { PHN vaccine } \\
\text { efficacy, \% } \\
\text { (range) }\end{array}$} & $\begin{array}{l}\text { RZV } \\
\text { Two doses }\end{array}$ & $98.4(95.0-100)$ & $98.4(95.0-100)$ & $98.4(95.0-100)$ & $97.84(94.1-100)$ & $97.84(94.1-100)$ \\
\hline & $\begin{array}{l}\text { RZV } \\
\text { One dose }\end{array}$ & $90.0(58.9-98.9)$ & $90.0(58.9-98.9)$ & $90.0(58.9-98.9)$ & $69.5(24.9-89.1)$ & $69.5(24.9-89.1)$ \\
\hline & ZVL & $69.8(30.8-89.6)$ & $65.69(25.4-84.2)$ & $65.69(25.4-84.2)$ & $73.38(51.6-85.8)$ & $39.51(0-73.8)$ \\
\hline
\end{tabular}

$\mathrm{HZ}$, herpes zoster; IC, immunocompromised; IC-free, immunocompetent; PHN, postherpetic neuralgia; RZV, adjuvanted recombinant zoster vaccine; YOA, years of age; ZVL, zoster vaccine live. 
the incidence rates of initial and recurrent $\mathrm{HZ}$ events are similar. ${ }^{229} 30$

\section{PHN probability}

PHN is defined as pain appearing or persisting for $>3$ months after initiation of HZ. PHN incidence was derived from published data. ${ }^{8}{ }^{31}$ Gauthier et al derived PHN incidence from the CPRD in the population excluding patients with underlying IC conditions using prescription medication records on top of PHN codes to identify these episodes. Forbes et al reported odds ratios of developing PHN for people with HIV and haematopoietic stem cell transplantation compared with IC-free population and these data were used in combination with data reported by Gauthier et al to model the proportion of PHN cases following an episode of $\mathrm{HZ}$ in the general population (table 1, online SI table 4). As for HZ, the model assumes that the incidence of recurrent PHN is the same as for first-time PHN.

\section{HZ-related mortality}

Values for HZ-associated mortality are based on published literature $^{32}$ (online SI table 5). The study by Edmunds $e t$ $a l$ was the only report including a granular breakdown of $\mathrm{HZ}$ case fatality rate by age group in the UK and was therefore considered to be the most appropriate source for HZ-associated mortality. The published data are based on the population of England and Wales. However, increasing mortality with increasing age is consistent with observations from studies conducted in other countries ${ }^{33}$ and it is assumed that these rates apply to the entire UK population.

\section{Non-PHN complications}

A wide range of complications other than PHN can occur in people experiencing an episode of $\mathrm{HZ}$ and could have a substantial impact on the burden of the disease. In the model, four main categories of complications were included, that is, ocular, neurological, cutaneous and other non-pain complications. Probabilities of developing these complications after the initial HZ episodes were taken from published literature ${ }^{29}$ (table 1).

\section{Hospitalisation and GP visits due to complications}

The CPRD study was used to derive the proportion of patients being hospitalised or visiting their GP due to HZ-related complications. ${ }^{28}$ Hospitalisation rates were higher in the IC cohort for all age groups. In addition, healthcare resource use was higher in older adults (online SI table 6 and 7).

\section{Vaccine efficacy and safety \\ Efficacy}

$\mathrm{VE}$ against $\mathrm{HZ}$ and $\mathrm{PHN}\left(\mathrm{VE}_{\mathrm{HZ}}\right.$ and $\mathrm{VE}_{\mathrm{PHN}}$, respectively) were derived from the SPS trial and the Zoster Efficacy and Safety Study for ZVL and from the ZOE-50 and ZOE-70 trials for RZV $\mathrm{RV}^{1623246}$ (table 1, online SI table 8). VE for RZV is based on a two-dose schedule given 2 months apart. However, compliance with second dose RZV is likely to be lower than $100 \%$, as such there is a cohort of individuals who are only vaccinated with one dose. Therefore, efficacy data for one-dose RZV were analysed post hoc based on limited clinical data from individuals in the ZOE trials receiving only one-dose $\mathrm{RZV}{ }^{25}$

Waning for both vaccines was modelled by linear fitting, using data from the above-mentioned trials as well as from the long-term persistence study (LTPS) for ZVL. ${ }^{25}$ For RZV, waning rates were assumed to be $1 \%$ (range: $0 \%-2.6 \%$ ) during the first 4 years after vaccination and $2.3 \%$ (range: $0.7 \%-4.6 \%$ ) thereafter in individuals $<70 \mathrm{YOA}$. In the population $\geq 70 \mathrm{YOA}$, waning rate was assumed to be constant over time at $3.6 \%$ (range: $1.4 \%-$ $6.6 \%) \cdot{ }^{25}$ For ZVL, the model indicated a waning rate of $5.4 \%$ (range: $4.5 \%-6.4 \%$ ) during the first 4 years after vaccination and $5.1 \%$ (range: $4.1 \%-6.0 \%$ ) thereafter in all age groups ${ }^{172}$ (online SI table 9).

\section{Coverage and compliance}

In the base-case analysis, coverage is set at $48.3 \%$ in line with latest coverage numbers for the UK. ${ }^{20}$ The impact of different coverage rates was assessed in sensitivity analyses. Compliance with the second dose of RZV was set to $70 \%$.

\section{Outcomes}

The model was used to estimate the avoidance of $\mathrm{HZ}$ and PHN cases, complications, deaths, GP visits and hospitalisations cases for three different vaccination strategies, that is, vaccination with RZV, vaccination with ZVL and no vaccination.

The number needed to vaccinate (NNV) to avert one case of $\mathrm{HZ}$ and PHN was also evaluated by applying the following calculation:

$$
\mathrm{NNV}=\frac{1}{\left(\frac{\text { control cases }}{\text { vaccinated persons }}\right)-\left(\frac{\text { vaccinated cases }}{\text { vaccinated persons }}\right)}
$$

\section{Scenario analyses}

Different scenario analyses were carried out where assumptions regarding vaccination coverage and compliance and age at vaccination were changed.

In a first scenario analysis, the impact of increasing coverage of RZV to $70 \%$ was explored. A higher coverage of $70 \%$ in the UK was deemed plausible considering that a) the influenza vaccine uptake in people $\geq 65 \mathrm{YOA}$ was $70.5 \%$ in $2016 / 2017^{34}$ and b) in the absence of a contraindication, vaccinators might not hesitate to administer the vaccine in IC individuals.

In a second scenario analysis, the second-dose compliance was varied, assuming a lower limit of $60 \%$ and an upper limit of $89 \%$ reflecting the lowest 10th percentile of the clinical trial second-dose compliance. ${ }^{25}$

Finally, the impact of changing the vaccination age on health outcomes was explored. VE is in general higher in younger individuals favouring early vaccination. On the other hand, duration of protection decreases over time and burden of disease (severity and duration of $\mathrm{HZ}$ and $\mathrm{PHN}$ ) is higher in older individuals, favouring vaccination 
at an older age. ${ }^{35}$ The relative balance of these factors may be different in case of ZVL and RZV, leading to different conclusions regarding optimal vaccination age.

\section{Sensitivity analyses}

Deterministic sensitivity analyses (DSA) were conducted to test the robustness of the results subject to changes in input parameters. To this aim, HZ and PHN incidence rates, VE and waning rates for both vaccines, incidence rate of HZ-related complications and vaccine-related adverse events, coverage and second-dose compliance were varied in one-way sensitivity analyses according to predefined ranges. Tornado diagrams were used to illustrate parameters that had the largest impact on $\mathrm{HZ}$ cases avoided.

Probabilistic sensitivity analysis (PSA) was carried out to assess the variability of results when changing parameters concomitantly using Monte Carlo simulation (5000 simulations). Each parameter could be attributed a value within its predefined range and according to the assigned probability distribution. A beta-distribution was used for all parameters except for vaccine coverage, which followed a uniform distribution. Age-specific incidence parameters which varied across age groups were assumed to be correlated using a correlation of 0.5 . The results of the PSA are presented using a histogram displaying the HZ cases avoided with RZV compared with ZVL.

\section{RESULTS}

\section{Base-case analysis}

In the base-case scenario (cohort $70 \mathrm{YOA}$ ), RZV reduced the number of HZ and PHN cases by 30262 and 5409, respectively, compared with no vaccination. ZVL led to a reduction of $7909 \mathrm{HZ}$ and $3567 \mathrm{PHN}$ cases (table 2). Vaccination with RZV reduced the number of HZ-related complications and the health-resource use (table 2). There were few HZ-related deaths; compared with no vaccination, RZV prevented eight HZ-related deaths while ZVL prevented none. The NNV to prevent one case of HZ was 12 with RZV and 45 with ZVL. The NNV to avoid one case of PHN was 65 with RZV and 98 with ZVL, respectively.

\section{Scenario analyses}

In a first scenario analysis, we increased coverage from $48.3 \%$ to $70 \%$ for RZV. In this scenario, an additional $13596 \mathrm{HZ}$ and $2430 \mathrm{PHN}$ cases would be prevented in the routine vaccination cohort ( $70 \mathrm{YOA}$ ) (figure 1 , light blue bar showing the additional proportion of $\mathrm{HZ}$ and $\mathrm{PHN}$ cases avoided with RZV compared with no vaccination).

In a second scenario analysis, compliance with second dose of RZV was set to lower and upper limits of $60 \%$ and $89 \%$, respectively. Compared with no vaccination, the numbers of $\mathrm{HZ}$ cases avoided with RZV were 28145 and 34284 at the lower and upper limits for compliance, respectively (figure 2 ).

To determine the optimal age for vaccination, scenario analyses were carried out to evaluate the public health impact in different age cohorts $(50,60,65,70$ and 80 YOA) in terms of NNV, HZ and PHN cases avoided and resource utilisation per 100000 people.

In case of RZV, the scenario that led to avoidance of the most HZ cases per 100000 people would be vaccinating at $60 \mathrm{YOA}$, while slightly more PHN cases per 100000 people could be avoided by vaccinating at 65 YOA. In case of ZVL, the number of HZ cases avoided per 100000

Table 2 Health outcomes and health resource utilisation in the vaccination cohort 70 YOA-base-case analysis, $n=722616$

\begin{tabular}{|c|c|c|c|c|c|}
\hline & $\mathbf{R Z V}$ & ZVL & No vaccination & $\begin{array}{l}\text { RZV vs no } \\
\text { vaccination }\end{array}$ & $\begin{array}{l}\text { ZVL vs no } \\
\text { vaccination }\end{array}$ \\
\hline $\mathrm{HZ}$ cases, $\mathrm{n}$ & 88643 & 110996 & 118905 & 30262 & 7909 \\
\hline PHN cases, $n$ & 16570 & 18411 & 21979 & 5409 & 3567 \\
\hline \multicolumn{6}{|l|}{ HZ-related complications } \\
\hline Total, $\mathrm{n}$ & 13109 & 16405 & 17565 & 4455 & 1160 \\
\hline Ocular, $n$ & 4207 & 5221 & 5548 & 1341 & 327 \\
\hline Neurological, $n$ & 4565 & 5782 & 6255 & 1691 & 474 \\
\hline Cutaneous, $\mathrm{n}$ & 2001 & 2492 & 2658 & 657 & 165 \\
\hline Other non-pain, $\mathrm{n}$ & 2336 & 2910 & 3103 & 767 & 193 \\
\hline \multicolumn{6}{|l|}{ Deaths } \\
\hline HZ-related deaths, $n$ & 56 & 64 & 64 & 8 & 0 \\
\hline \multicolumn{6}{|l|}{ Resource utilisation } \\
\hline Hospitalisation, $n$ & 7827 & 9463 & 9820 & 1993 & 357 \\
\hline GP visits, $n$ & 438328 & 546691 & 583612 & 145284 & 36921 \\
\hline
\end{tabular}

Coverage for both RZV and ZVL was set to $48.3 \%$. Second-dose compliance for RZV was set to $70 \%$.

GP, general practitioner; $\mathrm{HZ}$, herpes zoster; $n$, number of cases; PHN, postherpetic neuralgia; RZV, adjuvanted recombinant zoster vaccine;

YOA, years of age; ZVL, zoster vaccine live. 


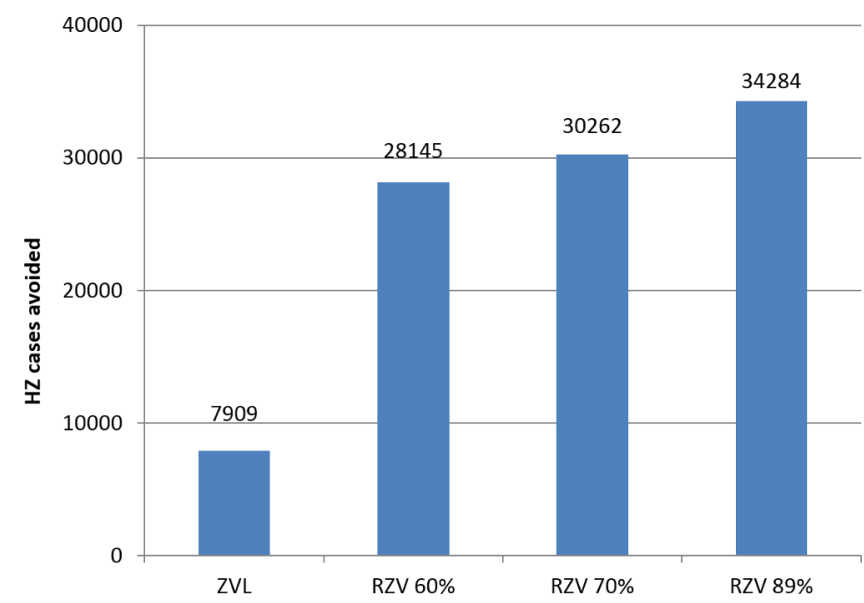

Figure 2 Impact of second-dose RZV compliance on $\mathrm{HZ}$ incidence. $\mathrm{HZ}$, herpes zoster; RZV, adjuvanted recombinant zoster vaccine; ZVL, zoster vaccine live.

people would be highest in the 65 YOA cohort, but more PHN cases per 100000 people would be avoided in the 70 YOA cohort (figure 3). In all age groups, number of $\mathrm{HZ}$ and PHN cases avoided per 100000 people was higher for RZV compared with ZVL. Complications avoided ranged from 689 with RZV and 250 with ZVL in the 65 YOA cohort, to 434 with RZV and 46 with ZVL in the 80 YOA cohort.

Consistent with these results, for RZV, the NNV to avoid one case of $\mathrm{HZ}$ was lowest in the $60 \mathrm{YOA}(\mathrm{NNV}=9)$ and the NNV to avoid one case of PHN was lowest in the 65 YOA cohort $(\mathrm{NNV}=54)$ (table 3$)$.

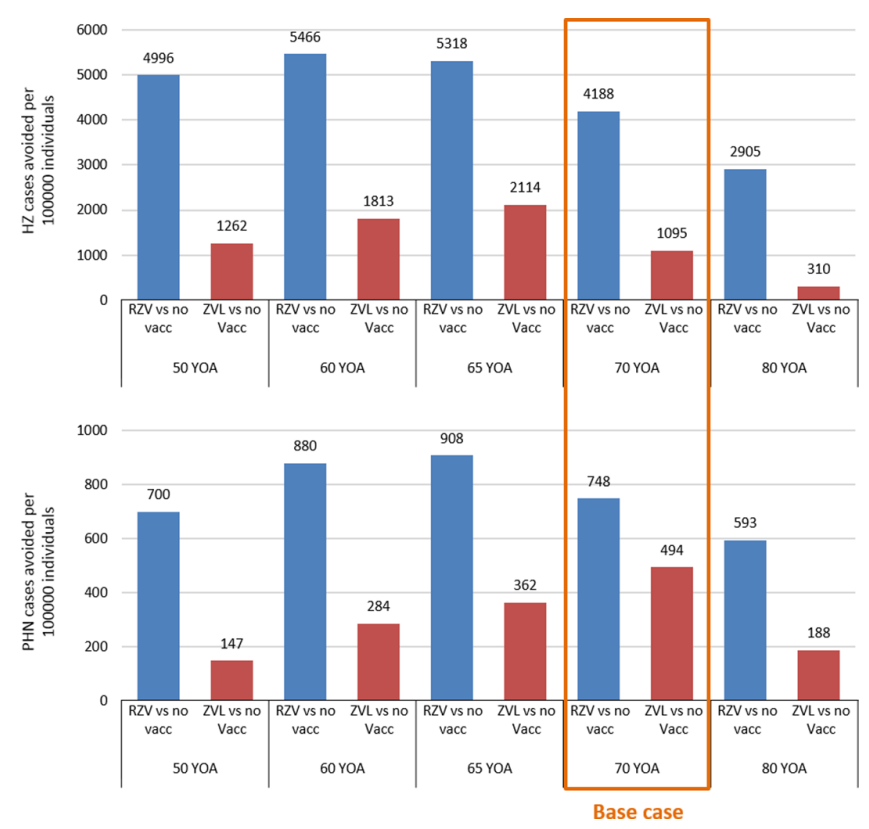

Figure 3 Scenario analyses: HZ (top) and PHN (down) cases avoided per 100000 individuals for different vaccination cohorts. Coverage for both RZV and ZVL was set to $48.3 \%$. Second-dose compliance for RZV was set to $70 \%$. $\mathrm{HZ}$, herpes zoster; no vac, no vaccination; PHN, postherpetic neuralgia; RZV, adjuvanted recombinant zoster vaccine; YOA, years of age; $Z \mathrm{VL}$, zoster vaccine live.
Table 3 NNV to avoid one case of $\mathrm{HZ}$ or PHN according to age at vaccination

\begin{tabular}{lcclll}
\hline & \multicolumn{2}{l}{$\mathbf{N N V ~ H Z}$} & & \multicolumn{2}{l}{ NNV PHN } \\
\cline { 2 - 3 } \cline { 5 - 6 } Age cohort & $\mathbf{R Z V}$ & $\mathbf{Z V L}$ & & RZV & ZVL \\
\hline 50 YOA & 10 & 39 & 69 & 328 \\
60 YOA & 9 & 27 & 55 & 171 \\
65 YOA & 10 & 23 & 54 & 134 \\
70 YOA & 12 & 45 & 65 & 98 \\
80 YOA & 17 & 156 & & 82 & 258 \\
\hline
\end{tabular}

Coverage for both RZV and ZVL was set to $48.3 \%$. Second-dose compliance for RZV was set to $70 \%$.

$\mathrm{HZ}$, herpes zoster; NNV, number needed to vaccinate; RZV, adjuvanted recombinant zoster vaccine; YOA, years of age; ZVL, zoster vaccine live.

The higher number of $\mathrm{HZ}$ and PHN cases avoided with RZV compared with ZVL across all age cohorts leads to an important reduction in the use of healthcare resources, which might be an indicator of a reduction in direct costs due to HZ (table 4). The number of GP visits per 100000 people avoided is highest for the $60 \mathrm{YOA}$ and $65 \mathrm{YOA}$ cohorts for both vaccines, and consistently higher for RZV compared with ZVL. The number of hospitalisations avoided increases with increasing age for RZV, reflecting the increased risk of hospitalisation due to $\mathrm{HZ}$ in older individuals.

\section{Sensitivity analyses}

In DSA analyses carried out for the base-case scenario in the age cohort $70 \mathrm{YOA}$, the robustness of results was tested by changing input parameters to their lower and upper estimated confidence ranges (online SI table 3-5; online SI table 8-9). In the base-case analyses, RZV prevented an additional $22353 \mathrm{HZ}$ cases as compared with ZVL. The parameter with the highest impact on the relative advantage of RZV over ZVL was annual waning of RZV (two doses) $\mathrm{VE}_{\mathrm{HZ}}$ in people $\geq 70 \mathrm{YOA}$, although the highest waning for RZV would still lead to a reduction of over $13000 \mathrm{HZ}$ cases compared with ZVL. Other parameters influencing the number of $\mathrm{HZ}$

Table 4 Reduction on resource utilisation per 100000 people

\begin{tabular}{llllll}
\hline & \multicolumn{2}{c}{ GP visits avoided } & & \multicolumn{2}{c}{ Hospitalisations avoided } \\
\cline { 2 - 3 } \cline { 6 - 6 } & $\mathbf{R Z V}$ & ZVL & & RZV & ZVL \\
\hline 50 YOA & 17481 & 3652 & & 126 & 17 \\
60 YOA & 22078 & 6375 & & 216 & 42 \\
65 YOA & 23447 & 8702 & & 266 & 69 \\
70 YOA & 20105 & 5109 & & 276 & 49 \\
80 YOA & 15243 & 1629 & 394 & 42 \\
\hline
\end{tabular}

Coverage for both RZV and ZVL was set to $48.3 \%$. Second-dose compliance for RZV was set to $70 \%$.

GP, general practitioner; $\mathrm{HZ}$, herpes zoster; RZV, adjuvanted recombinant zoster vaccine; YOA, years of age; ZVL, zoster vaccine live. 


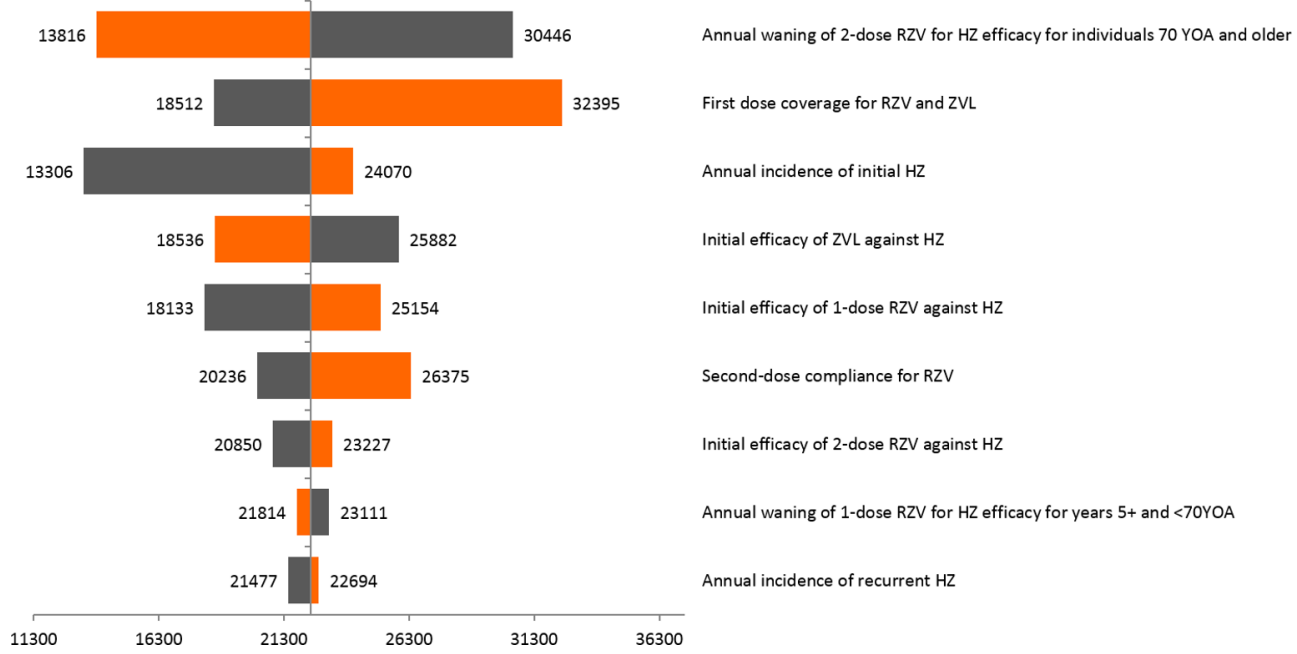

Figure 4 Tornado diagram: HZ cases avoided with RZV compared with ZVL-base-case analysis (70 YOA; coverage 48.3\%; compliance $70 \%$ ). Lower values are in orange and upper values are in grey. $\mathrm{HZ}$, herpes zoster; RZV, adjuvanted recombinant zoster vaccine; YOA, years of age; ZVL, zoster vaccine live.

cases avoided include initial $\mathrm{VE}_{\mathrm{HZ}}$ in people $\geq 70 \mathrm{YOA}$ for $\mathrm{ZVL}$ and RZV single dose, HZ incidence and RZV compliance to second dose (figure 4).

During PSA, all parameters were varied simultaneously along their predefined ranges. In all simulations $(\mathrm{n}=5000)$, RZV led to a reduction of $\mathrm{HZ}$ cases as compared with ZVL. The distribution of the number of $\mathrm{HZ}$ cases avoided by RZV relative to ZVL is shown in figure 5 . Overall, $83.1 \%$ of simulations predicted that RZV would prevent at least 15000 additional $\mathrm{HZ}$ cases compared with ZVL in the age group 70 YOA.

\section{DISCUSSION}

UMV against HZ using ZVL was introduced in the UK in 2013 and observational studies suggest that the programme has brought down HZ incidence by approximately one-third in the vaccinated cohorts. ${ }^{36} 37$ RZV has been approved by the EMA in individuals $\geq 50 \mathrm{YOA}$, thereby offering an alternative option to vaccinate people against HZ in addition to the existing ZVL. The aim of this study was to evaluate the public health impact of RZV in terms of $\mathrm{HZ}$ prevention compared with ZVL or no vaccination in the UK setting.

In the base case considering the current vaccination cohort of people $70 \mathrm{YOA}$, RZV reduced the number of HZ and PHN cases by 30262 and 5409 compared with no vaccination. In comparison, ZVL prevented $7909 \mathrm{HZ}$ and 3567 PHN cases as compared with no vaccination. $\mathrm{NNV}$ to prevent one episode of $\mathrm{HZ}$ was almost four times lower with RZV compared with RZV, that is, 12 with RZV vs 45 with ZVL. In addition, the estimated number of hospitalisations and GP visits due to $\mathrm{HZ}$ and PHN were substantially lower with RZV compared with HZ. HZ-related mortality is in general low; nevertheless, our simulations predicted that eight deaths could be prevented with a RZV vaccination strategy while no HZ-related deaths were prevented adopting a ZVL vaccination strategy.

Results were robust under DSA and PSA. Annual waning of RZV VE in people $\geq 70 \mathrm{YOA}$ had the greatest impact on the number of $\mathrm{HZ}$ avoided relative to $\mathrm{ZVL}$, but

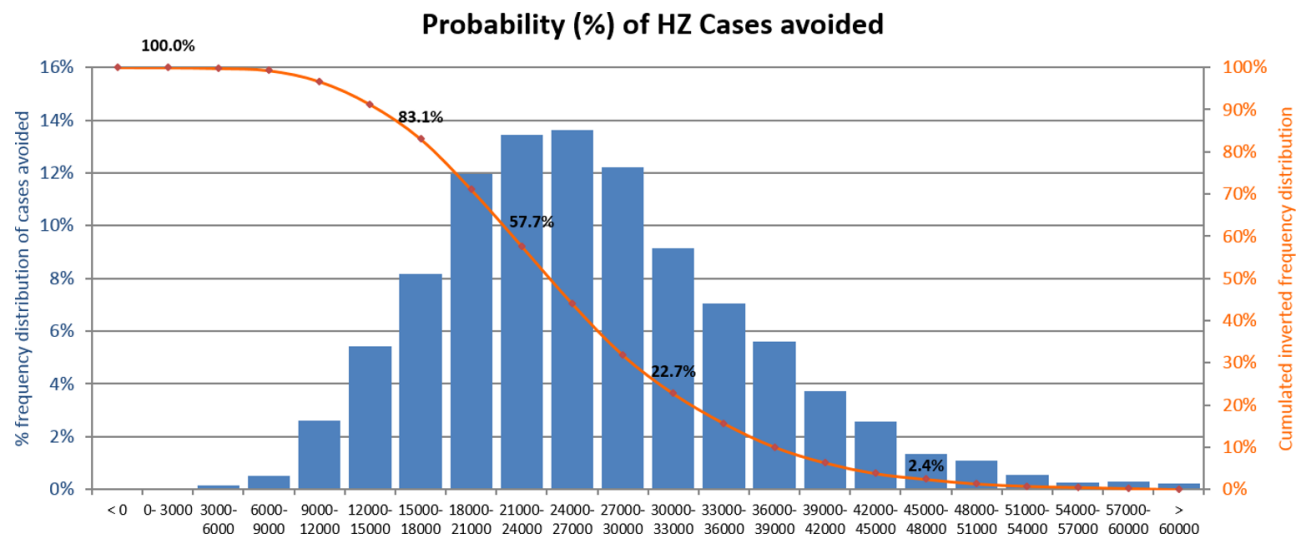

Figure 5 Probabilistic sensitivity analysis: HZ cases avoided with RZV compared with ZVL. The orange line shows the percentage of simulations averting at least the number of $\mathrm{HZ}$ cases shown on the $\mathrm{x}$-axis. $\mathrm{HZ}$, herpes zoster; RZV, adjuvanted recombinant zoster vaccine; ZVL, zoster vaccine live. 
even assuming an extreme assumption on waning, with an annual waning rate of $6.6 \%$, RZV would prevent an additional $13816 \mathrm{HZ}$ cases as compared with ZVL. Other parameters to which the relative vaccination strategies proved sensitive included annual $\mathrm{HZ}$ incidence and $\mathrm{VE}_{\mathrm{HZ}}$ of RZV and ZVL. PSA were always in favour of the RZV vaccination strategy with $83.1 \%$ of simulations showing a reduction of at least $\geq 15000 \mathrm{HZ}$ cases with respect to ZVL. We also tested different scenarios in which coverage and compliance were varied, assuming that the public health impact would increase as a greater proportion of individuals would be vaccinated. Increasing the coverage estimate of the first dose of RZV from $48.3 \%$ to $70 \%$ would further reduce $\mathrm{HZ}$ and PHN incidence, thereby leading to a greater reduction in healthcare resources used. We hypothesise that the coverage with RZV might be higher because a proportion of the eligible individuals are currently not receiving the vaccine with ZVL. Even though the proportion of individuals with a true contraindication to ZVL is estimated to be small $(2.8 \%),{ }^{20} \mathrm{HZ}$ vaccination with ZVL might be withheld even in those IC individuals who have no contraindications as vaccinators may have been risk averse. Reducing RZV compliance to $60 \%$, RZV would still prevent approximately three times more HZ cases compared with ZVL. This is in line with a recent public health impact study carried out for the German setting, where a compliance rate of $50 \%$ would still lead to an improvement of $200 \%$ over ZVL in terms of HZ prevention. ${ }^{25}$ Although results are in line with the German study, this UK model adaptation has some different methodological considerations that are of importance to potential decision-making bodies. First, this manuscript also assesses single-year cohorts versus multiple-year cohorts. This was chosen to reflect the current $\mathrm{HZ}$ vaccination programme in the UK where people get vaccinated with ZVL at $70 \mathrm{YOA}$ and $78 \mathrm{YOA}$ within the catch-up programme. Second, the HZ incidence is calculated based on a weighting method of IC-free and IC populations using the prevalence of IC in the different age groups. This is important to estimate the actual HZ incidence in the general population.

The recommended vaccination strategy was based on the clinical profile of $\mathrm{ZVL}$, the only vaccine available at the time. In its recommendation, the JCVI noted that ZVL VE decreases with increasing age and over time; hence, the current age cohort eligible for vaccination, that is, individuals $70 \mathrm{YOA}$, is a compromise to optimise limited efficacy and duration of protection against HZ. The JCVI also stated that optimal age at vaccination would depend on the characteristics of any given vaccine. ${ }^{38}$ Therefore, the impact of vaccination age on $\mathrm{HZ}$ and $\mathrm{PHN}$ incidence was explored through scenario analyses including different age cohorts (50, 60, 65, 70 and $80 \mathrm{YOA})$. The number of $\mathrm{HZ}$ and PHN cases avoided per 100000 people was higher with RZV than with ZVL across all age cohorts. In case of RZV, most HZ cases were avoided in the $60 \mathrm{YOA}$ cohort, while PHN case avoidance was highest in the 65 YOA cohort. This observation is consistent with a higher probability of developing
PHN at an increased age. On the other hand, the projected number of PHN cases avoided with ZVL was highest in the 70 YOA. This finding is due to a top-up efficacy seen with ZVL against PHN in the population $\geq 70$ YOA: vaccinated individuals with breakthrough $\mathrm{HZ}$ are at a lower risk of developing $\mathrm{PHN}$ as compared with unvaccinated individuals with HZ. In the individuals $<70 \mathrm{YOA}$, no additional protection against PHN was observed in clinical studies with ZVL. For RZV no additional top-up efficacy could be calculated based on the limited number of breakthrough cases, and thus $\mathrm{VE}_{\mathrm{HZ}}$ and $\mathrm{VE}_{\mathrm{PHN}}$ were assumed to be the same. As a result, for RZV, the NNV to avoid one case of $\mathrm{HZ}$ and PHN was lowest for the 60 YOA and 65 YOA cohorts. NNV increased in the 70 YOA and more so in the $80 \mathrm{YOA}$, where a proportion of the simulated cohort died due to natural causes before any health benefit of vaccination occurred.

From a healthcare utilisation perspective, RZV reduced the number of GP visits by $>13000$ compared with ZVL in all age groups. The highest reduction in GP visits was predicted in the 65 YOA cohort, while the largest impact on hospitalisations was predicted for the $80 \mathrm{YOA}$ cohort. The latter might be explained by the higher risk of hospitalisation inherent to older individuals due to a higher degree of frailty. Nevertheless, it should be noted that the reduction in hospitalisations was predicted to be several-fold higher with RZV compared with ZVL in all age cohorts. Reduction in the use of healthcare resources is a good indicator of potential decrease in direct costs of new healthcare interventions; however, this requires further investigation in a cost-effectiveness analysis with RZV in the UK context.

The potential public health impact of RZV in the UK setting has previously been studied by our group. ${ }^{39}$ The study showed a substantial reduction in HZ and PHN cases compared with no vaccination; however, no comparison was made to ZVL. A number of studies have evaluated the impact of ZVL on disease burden and associated cost-effectiveness in the UK setting. van Hoek et al analysed cost-effectiveness of ZVL in different age groups with the base-case considering a cohort of immunocompetent individuals aged 65 years in the UK. This cohort was modelled over a lifetime and a vaccine coverage of $73.5 \%$. Waning rates might have been underestimated in this model since long-term data from the LTPS study for persistence of efficacy of the ZVL vaccination were not yet taken into account. ${ }^{25}$ The LTPS study showed that $\mathrm{VE}_{\mathrm{HZ}}$ of ZVL decreases significantly over time with no statistically significant protection observed after 8 years of vaccination. ${ }^{17} 18$ In the economic model published by Moore $e t$ $a l$, the NNV of ZVL to prevent one case of $\mathrm{HZ}$ was 15 , and hence lower than that found in our simulations. However, the authors assumed a waning rate of $0 \% .^{10}$

The public health impact of RZV was also evaluated for other settings, including Germany, the USA, Canada and Australia. These studies used a wide range of assumptions regarding coverage, compliance and duration of vaccine protection for both RZV and ZVL. ${ }^{28}{ }^{40-42}$ Despite differences in these assumptions, all studies showed a consistent improvement in the reduction of $\mathrm{HZ}$ cases and its 
complications compared with no vaccination or vaccination with ZVL. In a recent independent cost-effectiveness study for the US setting, employing conservative assumptions regarding RZV waning rate, coverage and compliance, the authors concluded that RZV was more effective compared with ZVL under the vast majority of assumptions evaluated. ${ }^{40}$

As with every model, there are strengths and limitations associated with the modelling strategy employed. For RZV, most recent UK-specific data available at the time we conducted this study were used; for $\mathrm{HZ}$ incidence the CPRD database, a large UK-specific database, was analysed and values for both IC and non-IC cohorts were combined. ${ }^{28}{ }^{43}$ For PHN incidence, published data from two reports were used to estimate the PHN probability in the total population including individuals with immunodeficient states. The estimates of PHN cases prevented are close to real values, validating our approach. Demographic data projected to the year 2018 were used based on numbers reported by the ONS. ${ }^{27}$ The limitations in this study are related to assumptions that had to be made in the absence of real-world data, including coverage with RZV, compliance and long-term waning for RZV. Coverage and compliance were set to values observed in comparable vaccination programmes and these parameters were varied in scenario and one-way sensitivity analyses. Results from long-term studies with RZV are still outstanding and follow-up data are currently limited to 4 years. However, the model has been developed such that it can be updated once additional data become available. For ZVL waning rates, we included both data from the SPS and the LTPS study ${ }^{25}$ to ensure that we could compare ZVL and RZV in the ZONA model. Recent observational studies looking into the vaccine effectiveness of ZVL show that the vaccine wanes rapidly and has little to no protection left beyond year 8 after vaccination. ${ }^{18}{ }^{37}$ Finally, the rate of HZ-associated complications was assumed to be the same in all individuals with $\mathrm{HZ}$ regardless of their vaccination status. This assumption ignores the potential benefit vaccination might have by lowering the severity and duration of breakthrough $\mathrm{HZ}$ cases. Clinical trial data suggest that $\mathrm{VE}_{\mathrm{HZ}}$ and $\mathrm{VE}_{\mathrm{PHN}}$ are similar and there is some evidence that duration and severity of $\mathrm{HZ} / \mathrm{PHN}$ pain is lower in individuals having received RZV as compared with unvaccinated individuals. ${ }^{44}$

Future research might be directed towards assessing severity and duration of $\mathrm{HZ}$ and PHN cases depending on vaccination status, identifying subgroups of the population that may have enhanced benefit from the vaccine and evaluating cost-effectiveness in the current UMV cohort and across different age cohorts.

A lay language summary contextualising the outcomes and potential impact of this study for healthcare providers is displayed in figure 6 .

\section{CONCLUSION}

Within the model assumptions, RZV has the greater public health impact in terms of $\mathrm{HZ}$ and PHN case avoidance

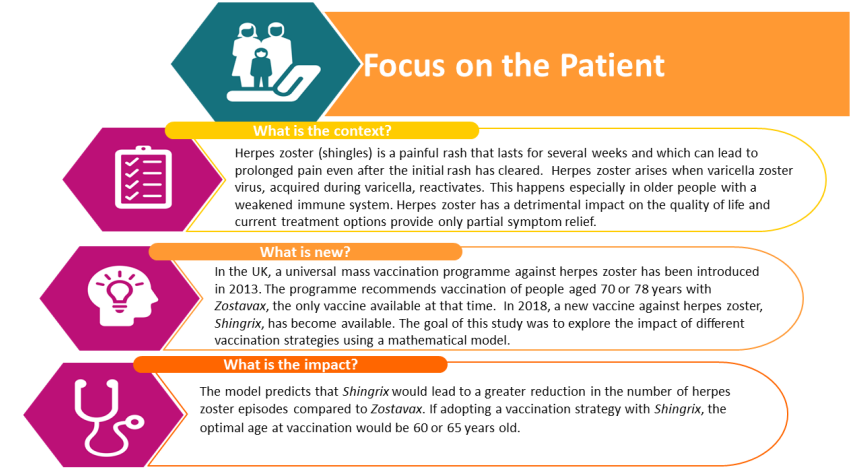

Figure 6 Lay language summary of the study.

and reduction in healthcare utilisation. When the UMV was introduced in 2013, vaccinating people at 70 YOA was the best option based on the vaccine characteristics of ZVL. With the approval of RZV in the USA, Canada, Japan and Europe in adults $\geq 50 \mathrm{YOA}$, the optimal HZ prevention strategy needs to be re-evaluated. The model projects for RZV a longer duration of protection and the VE remains high in older age groups compared with ZVL. Therefore, the results of this model show that the difference in clinical profile of RZV leads to a different optimal age of vaccination. Vaccinating the UK population with RZV at 60 YOA or 65 YOA is the optimal vaccination strategy in terms of public health impact, while being superior to ZVL in all age cohorts studied.

Acknowledgements The authors would like to thank Lijoy Varghese for his contribution to the study. The authors would like to thank Business \& Decision Life Sciences platform for editorial assistance and publications coordination, on behalf of GSK. Stephanie Garcia coordinated manuscript development and editorial support. Katrin Spiegel provided writing support.

Contributors DVO, DC, SL, BB participated to the conception and design of the analysis; DV0, DC, MH developed and adapted the model; DV0, DC, MH, HSC, $B B$ were involved in the collection, analysis and/or interpretation of the data. All authors had full access to the data and approved the final version of the paper for submission.

Funding GlaxoSmithKline Biologicals SA funded this study (GSK study identifier: H0-17-18511) and was involved in all stages of study conduct, including analysis of the data. GlaxoSmithKline Biologicals SA also covered all costs associated with the development and publication of this manuscript.

Competing interests DVO, MH, BB, DC and HSC are employees of the GSK group of companies. MH, DC and HSC hold shares in the GSK group of companies. SL is a freelance consultant working on behalf of the GSK group of companies.

Patient consent for publication Not required.

Ethics approval Ethical approval is not applicable for this public health impact modelling analysis.

Provenance and peer review Not commissioned; externally peer reviewed.

Data sharing statement All data used in this study are presented in the manuscript, references to the original material are provided. Please contact the corresponding author shall you require any additional information.

Author note Shingrix is a trademark owned by or licensed to the GSK group of companies. Zostavax is a trademark from Merck Sharp \& Dohme Corp.

Open access This is an open access article distributed in accordance with the Creative Commons Attribution Non Commercial (CC BY-NC 4.0) license, which permits others to distribute, remix, adapt, build upon this work non-commercially, and license their derivative works on different terms, provided the original work is properly cited, appropriate credit is given, any changes made indicated, and the use is non-commercial. See: http://creativecommons.org/licenses/by-nc/4.0/. 


\section{REFERENCES}

1. Mueller $\mathrm{NH}$, Gilden $\mathrm{DH}$, Cohrs RJ, et al. Varicella zoster virus infection: clinical features, molecular pathogenesis of disease, and latency. Neurol Clin 2008;26:675-97.

2. Kawai K, Gebremeskel BG, Acosta CJ. Systematic review of incidence and complications of herpes zoster: towards a global perspective. BMJ Open 2014;4:e004833

3. van Hoek AJ, Gay N, Melegaro A, et al. Estimating the costeffectiveness of vaccination against herpes zoster in England and Wales. Vaccine 2009;27:1454-67.

4. Johnson RW, Alvarez-Pasquin MJ, Bijl M, et al. Herpes zoster epidemiology, management, and disease and economic burden in Europe: a multidisciplinary perspective. Ther Adv Vaccines 2015;3:109-20.

5. Bollaerts K, Riera-Montes M, Heininger U, et al. A systematic review of varicella seroprevalence in European countries before universal childhood immunization: deriving incidence from seroprevalence data. Epidemiol Infect 2017;145:2666-77.

6. Brisson M, Edmunds WJ, Law B, et al. Epidemiology of varicella zoster virus infection in Canada and the United Kingdom. Epidemiol Infect 2001;127:305-14.

7. Pinchinat $\mathrm{S}$, Cebrián-Cuenca AM, Bricout $\mathrm{H}$, et al. Similar herpes zoster incidence across Europe: results from a systematic literature review. BMC Infect Dis 2013;13:170.

8. Gauthier A, Breuer J, Carrington D, et al. Epidemiology and cost of herpes zoster and post-herpetic neuralgia in the United Kingdom. Epidemiol Infect 2009;137:38-47.

9. Varghese L, Standaert B, Olivieri A, et al. The temporal impact of aging on the burden of herpes zoster. BMC Geriatr 2017;17:30.

10. Moore L, Remy V, Martin M, et al. A health economic model for evaluating a vaccine for the prevention of herpes zoster and postherpetic neuralgia in the UK. Cost Eff Resour Alloc 2010;8:7.

11. Gater A, Abetz-Webb L, Carroll S, et al. Burden of herpes zoster in the UK: findings from the zoster quality of life (ZQOL) study. BMC Infect Dis 2014;14:402.

12. Drolet M, Brisson M, Schmader KE, et al. The impact of herpes zoster and postherpetic neuralgia on health-related quality of life: a prospective study. CMAJ 2010;182:1731-6.

13. Schmader KE, Sloane R, Pieper C, et al. The impact of acute herpes zoster pain and discomfort on functional status and quality of life in older adults. Clin J Pain 2007;23:490-6.

14. Joint Committee on Vaccination and Immunisation. Joint Committee on Vaccination and Immunisation Statement on varicella and herpes zoster vaccines. $2010 \mathrm{http}$ ///webarchive.nationalarchives.gov.uk/ 20120907151317/http://www.dh.gov.uk/prod_consum_dh/groups/ dh_digitalassets/@dh/@ab/documents/digitalasset/dh_133599.pdf (Accessed 26 Jun 2018).

15. Merck Sharp Dohme Ltd. Zostavax Summary of Product Characteristics (SmPC). https://www.medicines.org.uk/emc/product/ 6101/smpc (Accessed 26 Jun 2018).

16. Oxman MN, Levin MJ, Johnson GR, et al. A Vaccine to Prevent Herpes Zoster and Postherpetic Neuralgia in Older Adults. NEnglJMed 2005;352:2271-84.

17. Morrison VA, Johnson GR, Schmader KE, et al. Long-term persistence of zoster vaccine efficacy. Clin Infect Dis 2015;60:900-9.

18. Tseng HF, Harpaz R, Luo Y, et al. Declining effectiveness of herpes zoster vaccine in adults aged $\geq 60$ years. $J$ Infect Dis 2016;213:1872-5.

19. Public Health England. Shingles (herpes zoster): the green book, chapter 28a, 2016. https://www.gov.uk/government/publications/ shingles-herpes-zoster-the-green-book-chapter-28a. (Accessed 26 Jun 2018).

20. Public Health England. Herpes zoster (shingles) immunisation programme: September 2016 to August 2017 Report for England. https://assets.publishing.service.gov.uk/government/uploads/ system/uploads/attachment_data/file/667636/Annual_shingles report_2016-2017_.pdf (Accessed 26 Jun 2018).

21. Chlibek R, Pauksens K, Rombo L, et al. Long-term immunogenicity and safety of an investigational herpes zoster subunit vaccine in older adults. Vaccine 2016;34:863-8.

22. GlaxoSmithKline Biologicals SA. Shingrix Summary of Product Characteristics (SmPC). http://www.ema.europa.eu/docs/en_GB/ document_library/EPAR_-_Product_Information/human/004336/ WC500246550.pdf (Accessed 26 Jun 2018).
23. Lal H, Cunningham AL, Godeaux $\mathrm{O}$, et al. Efficacy of an adjuvanted herpes zoster subunit vaccine in older adults. $N$ Engl J Med 2015;372:2087-96.

24. Cunningham AL, Lal H, Kovac M, et al. Efficacy of the Herpes Zoster Subunit Vaccine in Adults 70 Years of Age or Older. N Engl J Med 2016;375:1019-32.

25. Curran D, Van Oorschot D, Varghese L, et al. Assessment of the potential public health impact of Herpes Zoster vaccination in Germany. Hum Vaccin Immunother 2017;13:2213-21.

26. Schmader KE, Levin MJ, Gnann JW, et al. Efficacy, safety, and tolerability of herpes zoster vaccine in persons aged $50-59$ years. Clin Infect Dis 2012;54:922-8.

27. Office of National Statistics. based National population projections. 2014. https://www.ons.gov.uk/file?uri=/peoplepopulationandc ommunity/populationandmigration/populationprojections/datasets/ localauthoritiesinenglandtable2/2014based/table2.xls (Accessed 13 Jun 2018).

28. Yanni EA, Ferreira G, Guennec M, et al. Burden of herpes zoster in 16 selected immunocompromised populations in England: a cohort study in the Clinical Practice Research Datalink 2000-2012. BMJ Open 2018;8:e.020528:e020528.

29. Yawn BP, Saddier P, Wollan PC, et al. A population-based study of the incidence and complication rates of herpes zoster before zoster vaccine introduction. Mayo Clin Proc 2007;82:1341-9.

30. Yawn BP, Wollan PC, Kurland MJ, et al. Herpes zoster recurrences more frequent than previously reported. Mayo Clin Proc 2011;86:88-93.

31. Forbes HJ, Thomas SL, Smeeth L, et al. A systematic review and meta-analysis of risk factors for postherpetic neuralgia. Pain 2016;157:30-54.

32. Edmunds WJ, Brisson M, Rose JD. The epidemiology of herpes zoster and potential cost-effectiveness of vaccination in England and Wales. Vaccine 2001;19(23-24):3076-90.

33. Bricout $\mathrm{H}$, Haugh $\mathrm{M}$, Olatunde $\mathrm{O}$, et al. Herpes zoster-associated mortality in Europe: a systematic review. BMC Public Health 2015;15:466.

34. Public Health England. Influenza: the green book, chapter 19. https:// assets.publishing.service.gov.uk/government/uploads/system/ uploads/attachment_data/file/663694/Greenbook_chapter_19 Influenza_.pdf (Accessed 29 Jun 2018).

35. Sampathkumar P, Drage LA, Martin DP. Herpes zoster (shingles) and postherpetic neuralgia. Mayo Clin Proc 2009;84:274-80.

36. Amirthalingam G, Andrews N, Keel P, et al. Evaluation of the effect of the herpes zoster vaccination programme 3 years after its introduction in England: a population-based study. Lancet Public Health 2018;3:e82-e90.

37. Walker JL, Andrews NJ, Amirthalingam G, et al. Effectiveness of herpes zoster vaccination in an older United Kingdom population. Vaccine 2018;36:2371-7.

38. Joint Committee on Vaccination Immunisation. Minute of the meeting on 04 October 2017. 2017 https://www.jostrust.org.uk/sites/ default/files/minute_2017_10_draft.pdf (Accessed 25 Mar 2019).

39. van Oorschot DA, Hunjan M, Varghese L, et al. The public health perspective of an investigational herpes zoster vaccine in the United Kingdom (UK). Value in Health 2016;19:A400-A00.

40. Le P, Rothberg MB. Cost-effectiveness of the adjuvanted herpes zoster subunit vaccine in older adults. JAMA Intern Med 2018;178:248-58.

41. Varghese L, Curran D, Yan S, et al. Estimating the Potential Public Health Impact of Introducing the HZ/su Vaccine in the US Population Aged $\geq 50$ Years. Open Forum Infect Dis 2015;2.

42. Varghese L, Nissen M, Olivieri A, et al. Public health perspective of phase III results of an investigational Herpes Zoster vaccine. Public Health Association of Australia. Public Health Association of Australia - Communicable Disease Control Conference; 1-2 June 2015, Brisbane, Australia, 2015:42. https://www.phaa.net.au/documents/ item/581

43. Curran D, Hunjan M, El Ghachi A, et al. Herpes zoster related healthcare burden and costs in both immunocompromised (ic) and ic-free populations in the United Kingdom. Value in Health 2017;20:A786.

44. Curran D, Athan E, Diez-Domingo J, et al. Quality-of-life impact of an investigational subunit-adjuvanted herpes zoster vaccine in adults $\geq 50$ years of age. Open Forum Infect Dis 2016;3. 